\title{
Mitochondrial DNA and IQ in Europe
}

\author{
MARK G. THOMAS \\ University College London \\ KEVIN W.P. MILLER \\ University of California at Santa Barbara \\ C.G.N. MASCIE-TAYLOR \\ University of Cambridge
}

\begin{abstract}
Recently a mitochondrial DNA polymorphism (EST00083) was found at significantly different frequencies in high IQ and low IQ groups in two independent studies. We have used mitochondrial sequences from a range of populations to show that this polymorphism occurred more than once in human history. Furthermore, the polymorphism is particularly common in Europe where it is predominantly associated with a single mitochondrial line (lineage) that appears to date back to the expansion of anatomically modern humans into Europe about 35,000 years ago. Examination of the genetic diversity within this mitochondrial lineage, together with the known migration and settlement of Europeans into the USA, suggests that the observed IQ associated polymorphism found in Cleveland Ohio is likely to be restricted to only one form (sub-group) of this mitochondrial lineage.
\end{abstract}

Family, twin, and adoption data indicate that approximately half of the variance associated with IQ may be due to genetic factors (Bouchard, Lykken, Mcgue, Segal, \& Tellegen, 1990; Chipuer, Rovine, \& Plomin 1990). In recent years the search for genetic markers (often called quantitative trait loci or QTL; Gelderman, 1975) associated with IQ has been aided by the development of molecular genetic techniques and, in particular, the method of allelic association (Owen \& McGuffin, 1993). The IQ QTL project (Plomin et al., 1994) used this approach when looking for differences in the frequency distribution of DNA markers occurring in or near genes that are likely to play a part in neural functioning. Using high- and low-IQ groups of Caucasian children from the greater Cleveland, Ohio metropol-

Direct all correspondence to: Mark G. Thomas, The Center for Genetic Anthropology, Departments of Biology and Anthropology, Darwin Building, University College London, Gower Street, London WC1E 6BT, England.

INTELLIGENCE 26(2): 167-173

ISSN: 0160-2896
Copyright $(C) 1998$ by Ablex Publishing Corporation All rights of reproduction in any form reserved. 
itan area, 100 polymorphic markers were surveyed for allelic association (Plomin et al., 1994; Plomin et al., 1995). Of these, eleven showed significant differences in frequency between the low- and high-IQ groups (twice the number expected by chance). However, in an unrelated replication sample only one of these polymorphic markers (EST00083) showed a significant association. Further investigation (Skuder et al., 1995) revealed that this marker is a mitochondrial polymorphism, resulting from the loss of a $M s p I$ restriction site (MspI-) at position 15,925 of the mitochondrial genome (Anderson et al., 1981). Apart from obvious questions relating to the mechanism by which a mitochondrial polymorphism may show a relationship with IQ score, the presence of such an association also raises some population genetics issues.

The inheritance of Mitochondrial DNA (mtDNA) is exclusively maternal because the mitochondria are located in the cytoplasm and mature sperm contain very little cytoplasm with few mitochondria. In addition, the mitochondrial genome is not generally believed to undergo intragenic recombination and as such can be considered as a totally linked genetic system. In the absence of recombination, a causative link can only be made between a mitochondrial polymorphism and a phenotypic character such as IQ if either; (a) a considerable amount of functional information exists about both the mutation and the measured phenotypic character, or (b) the polymorphism has arisen independently in several different mitochondrial lines (lineages) and is associated with the measured phenotypic character in all lineages.

The 16,569 base-pair (bp) mammalian mitochondrial genome encodes two ribosomal RNA genes, 22 transfer RNAs (tRNAs) and 13 polypeptides that are parts of multi-subunit enzymes involved in cellular energy metabolism (oxidative phosphorylation). The noncoding sequence is confined to a $1,122 \mathrm{bp}$ region known as the control region (CR). This region is the most variable part of the mitochondrial genome and is phylogenetically informative when comparing closely related maternal lineages such as those within our own species (Ward, Frazier, Dewjager, \& Paabo, 1991; Richards et al., 1996).

We have investigated the CR sequences associated with the MspI-individuals from a number of geographically distinct populations in order to address the following questions: (a) has this polymorphism occurred more than once in human history? (b) does this polymorphism show an uneven distribution between populations? (c) does this polymorphism show an association with any other mitochondrial DNA (mtDNA) markers, and (d) how old are the mtDNA lineage(s) that contain this polymorphism. Our sample comprises 144 individuals from the British Isles, 65 individuals from Island Southeast Asia and the Pacific, 7 individuals from France and 48 individuals from Mongolia.

\section{METHOD}

The European CR sequences are described in Miller (1996) and for Southeast Asian and Pacific DNA samples in Flint, Boyce, Martinson, \& Clegg, (1989). DNA was extracted from the blood of Mongolian individuals using the method of Boom et al (1990). Full details of the DNA extraction, PCR amplification and sequencing can be obtained from the first author. Phylogenetic trees were constructed, and the age of common nodes (in mutational time) were estimated using the two-cluster test (Takezaki, Rzhetsky, \& Nei, 1995). In this method, the age of a common node is calculated to be the average genetic distance between all sequence pairs on a phylogenetic tree that are connected by that common node. 
Genetic distances were estimated using the nucleotide substitution model of Tamura and Nei (1993), which assumes different mutation rates for different types of substitution, and compensates for unequal base compositions. To correct for substitution rate heterogeneity at different sites in the CR, a gamma correction was employed. A gamma parameter of 0.47 , a value previously estimated for human CR sequences (Wakeley, 1993), was used. Mutational time estimates were converted into real time estimates by assuming a CR nucleotide divergence rate of 33\% per million years (Ward et al., 1991).

\section{RESULTS}

Of the total sample, 12 individuals were found to have the $M s p I$ - polymorphism, of which 10 were from Europe and 2 were from Mongolia. The frequency of $\mathrm{MspI}$ - in our European populations $(8.3 \%)$ is just significantly lower $\left(\chi_{(d f=1)}^{2}=3.92 ; p<0.05\right)$ than the $14 \%$ found in the combined high-, medium- and low- IQ sample (Plomin et al. 1995). Other researchers have found the $M s p I$ - polymorphism present at a frequency of about $12 \%$ in Europe (L. Hill, personal communication). Thus it is likely that the lower frequency in our sample occurs because most of our European sample originated from the British Isles. Alternatively the frequency difference may reflect the effects of random genetic drift during the settlement of the USA by European populations.

However, the $12 \mathrm{MspI}$ - individuals do not have the same CR sequence. Comparison of their Control Regions (Figure 1) reveal that all of the European sequences have a $\mathrm{T}$ to $\mathrm{C}$

$\begin{array}{llll}111111111111111111111 & \text { E } & M \\ 666666666666666666666 & \text { Y } & \text { O } \\ 001111111222222223333 & \text { r } & n \\ 450244688456799990023 & & \\ 017607369370846784672 & & \end{array}$

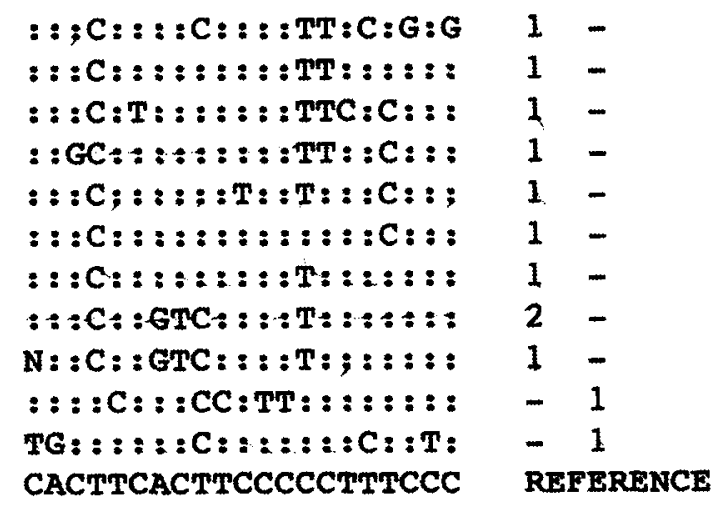

Figure 1. Control region sequences for $12 \mathrm{Mspl}$ - individuals. Sequence is numbered according to the reference sequence (Anderson et al., 1981) and was obtained between positions 16,024 and 16,400. Only sites that differ from the reference sequence are shown. 'Eur' and 'Mon' indicate European andMongolian individuals respectively. 
transition at position 16,126 while neither of Mongolian $M s p I$ - individuals show this change. In addition, nine out of the ten Europeans have a $\mathrm{C}$ to $\mathrm{T}$ transition at position 16,294. In a recent survey of European mtDNA-CR sequences these two transitions were found to be diagnostic for a major lineage group (group 2B) that is though to have expanded in Europe with the arrival of the first modern humans about 35,000 years ago (Richards et al., 1996). It is thought that this lineage group derived from the most common mtDNA type (haplotype) in Europe which had a CR sequence between positions 16,024 and 16,400 that is identical to the reference sequence (Anderson et al., 1981). Moreover, the $\mathrm{T}$ to $\mathrm{C}$ transition at position 16,126 most likely preceded the $\mathrm{C}$ to $\mathrm{T}$ transition at position 16,294 . However, Richards et al., (1996) have also suggested that group 2B forms only one branch of a broader lineage group (group 2) and that a second branch (group 2A; which is characterized by $T$ to $C$ transitions at positions 16,126 and 16,069 ) is also common throughout Europe.

Many of the CR sequences for our European samples were already known (Miller, 1996) and it was found that of the 16 samples containing $T$ to $C$ transitions at positions 16,126 and 16,069 , none had the $M s p I-(15,925)$ polymorphism. If one assumes the one European sample containing a transition at position 16,126 , but not at 16,294 , is not the result of a back mutation at position 16,294, then our data suggest that the European MspIlineage arose after the formation of lineage group 2 but before the formation of group $2 \mathrm{~B}$ (Richards et al., 1996).

We have estimated the age of the common node for our European $M s p I$ - CR sequences to be 37,000 years (SE 17.6\%). This is in close agreement with the estimated age for lineage group 2B (Richards et al., 1996) and coincides with the date for the colonization of Europe by modern humans (Lahr \& Foley, 1994). These sequences clustered with our 16 MspI+ CR sequences that contained transitions at positions 16,069 and 16,126 to form a lineage group that excluded the two Mongolian MspI- CR sequences and was connected to a common node dating to 60,000 years bp. The common node connecting all MspIsequences dates to 80,000 years where as the common node connecting the two Mongolian CR sequences dates to around 68,000 years. Richards et al., (1996) have shown lineage group 2B to be distributed evenly in Europe at a frequency of about $8 \%$ although it was not found in Finland $(n=29)$, Turkey $(n=22)$ or Iceland $(n=14)$. They have postulated that this group arose from an ancestral haplotype containing a transition at position 16,126 and that this haplotype originated, and is still common in the Middle East but not elsewhere. Taken together these data strongly suggest that the $M s p I$ - polymorphism arose independently in Europe and Mongolia.

\section{Discussion}

With the identification of highly polymorphic markers throughout the human genome, both in nuclear and mitochondrial DNA, it has become possible to search for genes which are responsible for some common multifactorial traits or for susceptibility for common multifactorial diseases. Two main types of analysis are used, either association or linkage analysis. Association studies seek to find the correlated occurrence of specific alleles at a genetic locus and the disease (or trait) in a population. In contrast, linkage studies search for the co-transmission, in families, of a marker locus (regardless of the specific allele at that locus), with the disease or trait. 
Plomin and colleagues $(1994,1995)$ made use of the association method when examining the relationship between 100 polymorphic markers and high and low IQ groups from Cleveland, Ohio. They found significantly more associations than expected by chance alone while a separate study revealed an association with only one polymorphic marker, EST00083. This marker was later shown (Skuder et al., 1995) to be a mitochondrial polymorphism. The entire 16,569 base-long mitochondrial chromosome sequence is known (Anderson et al., 1981) and this polymorphic marker results from the loss of a MspI restriction site (Mspl-) at position 15,925 of the mitochondrial genome.

The presence of a MspI- mutation has been previously demonstrated in Caucasian (Johnson, Wallace, Ferris, Rattazzi, \& Cavalli-Sforza, 1983; Santachiara Benerecetti, Scozzari, Semino, Torroni, Brega, \& Wallace, 1988; Benedictis, Rose, Passarino, \& Quagliariello, 1989; Semino, Torroni, Scozzari, Brega, \& Santachiara Benerecetti, 1991) and Oriental (Johnson et al., 1983; Semino et al., 1991) populations but $\mathrm{Mspl}$ - has not been found in a sub-Saharan African sample (Johnson et al., 1983). There is good reason to suspect that the Caucasian and Oriental MspI- mutations are not the same since the above researchers have also found differences between the $M s p I$ - banding patterns. Our results also suggest Caucasian and Oriental $M s p I$ - haplotypes differ in origin. In addition more than one polymorphism has been found that results in the loss of the $M s p I$ site at position 15,925 (Hill, personal communication).

Considering the central role that mtDNA gene products play in the energy metabolism of the cell, a causative link between mtDNA mutations and cognitive ability remains a strong possibility. Apart from the existence of a number of neuro-degenerative diseases associated with mtDNA lesions (Wallace, 1992; Shoffner \& Wallace, 1994), there is a case in which a patient's IQ was found to increase by 20 points upon treatment for mitochondrial dysfunction (Anezaki, Yanagisawa, Ibayashi, \& Miyatake, 1992).

Assuming that most of children used in the original IQ-QTL study (Plomin et al., 1994) are of European maternal ancestry, our work suggests that the MspI- individuals belong to an mtDNA lineage previously labeled 2B (Richards et al., 1996). Consequently if there is an mtDNA polymorphism that is causally linked with IQ in their sample, then it could occur anywhere on the mtDNA molecule and need only be associated with the $2 B$ lineage in order to explain the results of Skuder and co-workers (1995). As a result, the ability or inability to detect an association between IQ and mtDNA will depend upon which mitochondrial line(s) (lineage(s)) occurs in the study population. Failure to corroborate the results of Plomin et al (1994) might simply be due to the presence of a different MspI- mitochondrial lineage or sub-lineage. Thus there is no reason to expect that associations observed in one population should also occur in another population if the populations have different origins. Furthermore, because the $\mathrm{Mspl}$ - polymorphism has persisted in Europe for approximately 35,000 years and accounts for about $14 \%$ of European mtDNA haplotypes it seems unlikely that it is directly associated with a strong selective disadvantage. For this reason we postulate that the $M s p I$ - polymorphism is not itself causally linked with IQ but that a putative polymorphism causing low IQ in the original Cleveland sample (Plomin et al., 1994) occurs elsewhere on the mitochondrial genome and in only a sub-set of the MspI-haplotypes in that population.

The diversity of $M s p I$ - haplotypes in the original Cleveland sample (Plomin et al., 1994) has yet to be reported. Given that founder effects would have reduced genetic diversity and affected the distribution of $M s p I$ - haplotypes, it remains a strong possibility that 
many of the MspI- individuals in this sample belong to a sub-lineage that includes a mitochondrial polymorphism that causes low IQ. We suggest that sequencing of the mtDNA $\mathrm{CR}$ in MspI- individuals from the original Cleveland sample would prove invaluable in identifying such a polymorphism. Such an approach would prove extremely useful in any QTL project in which a link is suspected between mitochondrial mutations and phenotype.

Acknowledgements: We thank S. Hill, D. Ball and the anonymous referees for their comments on the manuscript. We thank J.J. Martinson and J.B. Clegg for the use of DNA samples from Pacific populations.

\section{REFERENCES}

Anderson, S., Bankier, A.T., Barrell, B.G., de Bruijn, M.H., Coulson, A.R., Drouin, J., Eperon, I.C., Nierlich, D.P., Roe, B.A., Sanger, F., Schreier, P.H., Smith, A.J., Staden, R. \& Young, I.G. (1981). Sequence and organization of the human mitochondrial genome. Nature, 290, 457-465.

Anezaki, T., Yanagisawa, K., Ibayashi, K., \& Miyatake, T. (1992). A patient with severe iron-deficiency anemia and memory disturbance. Internal Medicine, 31 (11), 1306-1309.

Benedictis, G.D., Rose, G., Passarino, G., \& Quagliariello, C. (1989). Restriction fragment length polymorphism of human mitochondrial DNA in a sample population from Apulia (Southern Italy). Annals Human Genetics, 53, 311-318.

Boom, R., Sol, C.J.A., Salimans, M.M.M., Jansen, C.L., Wertheim-Van Dillen, P.M.E., and van der Noorda, J. (1990). Rapid and simple method for purification of nucleic acids. Journal of Clinical Microbiology 28(3), 495-503.

Bouchard, T.J., Lykken, D.T., Mcgue, M., Segal, N.L., \& Tellegen, A. (1990). Sources of human psychological differences - the Minnesota study of twins reared apart. Science, 250(4978), 223-228.

Chipuer, H.M., Rovine, M., \& Plomin, R. (1990). LISREL modelling: Genetic and enviromental influences on IQ revisited. Intelligence, 14, 11-29.

Flint, J., Boyce, A.J., Martinson, J.J., \& Clegg, J.B. (1989). Population bottlenecks in polynesia revealed by minisatellites. Human Genetics, 83(3), 257-263.

Gelderman, H. (1975). Investigations on inheritance of quantitave characters in animals by gene markers. I, Methods. Theor. Appl. Genet., 46, 319-330.

Johnson, M.J., Wallace, D.C., Ferris, S.D., Rattazzi, M.C., \& Cavalli-Sforza, L.L. (1983). Radiation of human mitochondrial DNA types analyzed by restriction endonuclease cleavage patterns. J. Mol. Evol., 19, 255271.

Lahr, M.M., \& Foley, R.A. (1994). Multiple dispersals and modern human origins. Evolutionary Anthropology, 3, 48-61.

Miller, K.W.P. (1996). Molecular Genetic Analysis of Human Populations in Orkney and the North Atlantic Region. PhD Thesis, University of Cambridge.

Owen, M.J., \& McGuffin, P. (1993). Association and linkage: Complimentary strategies for complex disorders. Journal of Medical Genetics, 30, 638-639.

Plomin, R., McClearn, G.E., Smith, D.L., Vignetti, S., Chorney, M.J., Chorney, K., Venditti, C.P., Kasarda, S., Thompson, L.A., Detterman, D.K., Daniels, J., Owen, M., \& McGuffin, P. (1994). DNA markers associated with high versus low IQ- the IQ quantitative trait loci (qtl) project. Behavior Genetics, 24(2), 107118.

Plomin, R., Mcclearn, G.E., Smith, D.L., Skuder, P., Vignetti, S., Chorney, M.J., Chorney, K., Kasarda, S., Thompson, L.A., Detterman, D.K., Petrill, S.A., Daniels, J., Owen, M.J., \& McGuffin, P. (1995). Allelic associations between 100 DNA markers and high versus low IQ. Intelligence, 21(1), 31-48.

Richards, M., Cortereal, H., Forster, P., Macaulay, V., Wilkinsonherbots, H., demaine, A., Papiha, S., Hedges, R., Bandelt, H.J., \& Sykes, B. (1996). Paleolithic and neolithic lineages in the european mitochondrial gene pool. American Journal of Human Genetics, 59(1), 185-203.

Santachiara Benerecetti, A.S., Scozzari, R., Semino, O., Torroni, A., Brega, A., \& Wallace, D. C. (1988). Mitochondrial DNA polymorphisms in Italy. Annals Human Genetics, 52, 39-56. 
Semino, O., Torroni, A., Scozzari, R., Brega, A., \& Santachiara Benerecetti, A.S. (1991). Mitochondrial DNA polymorphisms among Hindus: A comparison with the Tharus of Nepal. Annals Human Genetics, 55 , 123-136.

Shoffner, J.M., \& Wallace, D.C. (1994). Oxidative-phosphorylation diseases and mitochondrial-dna mutationsdiagnosis and treatment. Annual Review of Nutrition, 14, 535-568.

Skuder, P., Plomin, R., Mcclearn, G.E., Smith, D.L., Vignetti, S., Chorney, M.J., Chorney, K., Kasarda, S., Thompson, L.A., Detterman, D.K., Petrill, S.A., Daniels, J., Owen, M.J., \& McGuffin, P. (1995). A polymorphism in mitochondrial-DNA associated with IQ. Intelligence, 21(1), 1-11.

Takezaki, N., Rzhetsky, A.,\& Nei, M. (1995). Phylogenetic test of the molecular clock and linearized trees. Molecular Biology and Evolution, 12(5), 823-833.

Tamura, K., \& Nei, M. (1993). Estimation of the number of nucleotide substitutions in the control region of mitochondrial DNA in humans and chimpanzees. Mol. Biol. Evol., 10(3), 512-526.

Wakeley, J. (1993). Substitution rate variation among sites in hypervariable region 1 of Human mitochondria! DNA. J. Mol. Evol., 37, 613-623.

Wallace, D.C. (1992). Diseases of the mitochondrial-DNA. Annual Review of Biochemistry, 61, 1175-1212.

Ward, R.H., Frazier, B.L., Dewjager, K., \& Paabo, S. (1991). Extensive mitochondrial diversity within a single amerindian tribe. Proceedings of The National Academy of Sciences of The United States of America, 88(19), 8720-8724. 